\title{
Is There a Spectral Theory for All Bounded Linear Operators?
}

\author{
Longyun Ding and Su Gao
}

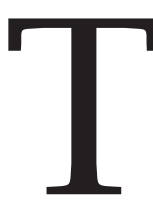

he answer is not known. But results from recent (and some not so recent) research suggest that there might be one, and in fact there likely is one. We will explain below what has been achieved and why it promises some more exciting research ahead.

\section{What Is Spectral Theory?}

By spectral theory we mean the theory of structure of certain bounded linear operators on a Hilbert space. In a broader sense, the history of spectral theory goes way back to the nineteenth century, when the objects of study used to be infinite systems of linear equations and integral equations. The subject was revolutionized in the late 1920s by von Neumann, when he defined the notion of an abstract Hilbert space and considered bounded linear operators on it. In this modern sense a successful spectral theory was soon obtained by Riesz for all compact operators as a direct extension of the theory of finite square matrices. By the early 1930s, von Neumann had obtained a satisfactory spectral theory for all normal operators, with self adjoint (or Hermitian) operators and unitary operators as important special cases.

Spectral theory has evolved further since then; a vast amount of work was done on extending spectral theory to various Banach algebras. However, efforts to extend spectral theory to all bounded linear operators on a Hilbert space have met with resistance so far. This will be the problem we concentrate on in this article. To be explicit, the problem we consider is the existence of a

Longyun Ding is professor of mathematics at Nankai University, China. His email address is ding1ongyun@gmai 1 . com.

Su Gao is professor of mathematics at the University of North Texas. His email address is su.gao@unt. edu.

DOI: http://dx.doi.org/10.1090/noti1146 satisfactory spectral theory for all bounded linear operators.

At this point we have to include a disclaimer: neither of the authors considers himself a functional analyst; in fact, both of us work in descriptive set theory, which is the study of definable sets of real numbers. So what does descriptive set theory have to do with this important problem in functional analysis? The answer is, we hope, that it provides some new perspectives on this old problem.

We employ some notation before continuing our discussion. Let $\mathcal{H}$ denote a separable infinitedimensional complex Hilbert space and let $B(\mathcal{H})$ denote the space of all bounded linear operators on $\mathcal{H}$. Let $N(\mathcal{H}), S(\mathcal{H})$, and $U(\mathcal{H})$ denote, respectively, the spaces of all normal, self adjoint, and unitary operators on $\mathcal{H}$. In notation,

$$
\begin{aligned}
N(\mathcal{H}) & =\left\{T \in B(\mathcal{H}): T T^{*}=T^{*} T\right\}, \\
S(\mathcal{H}) & =\left\{T \in B(\mathcal{H}): T=T^{*}\right\}, \\
U(\mathcal{H}) & =\left\{T \in B(\mathcal{H}): T T^{*}=T^{*} T=I\right\} .
\end{aligned}
$$

Obviously, $S(\mathcal{H})$ and $U(\mathcal{H})$ are subspaces of $N(\mathcal{H})$.

In a nutshell, here is how spectral theory works for a normal operator $T$. The spectrum of $T$, denoted by $\sigma(T)$, is the set of $\alpha \in \mathbb{C}$ such that $T-\alpha I$ is not invertible. If $\mathcal{B}$ is the $\sigma$-algebra of all Borel subsets of $\sigma(T)$, a spectral measure for $\sigma(T)$ is a function $E: \mathcal{B} \rightarrow B(\mathcal{H})$ such that

(a) $E(X)$ is a projection of $\mathcal{H}$ for every $X \in \mathcal{B}$;

(b) $E(\varnothing)=0$ and $E(\sigma(T))=1$;

(c) $E(X \cap Y)=E(X) E(Y)$ for $X, Y \in \mathcal{B}$;

(d) if $\left\{X_{n}\right\}_{n=1}^{\infty}$ is a collection of pairwise disjoint Borel sets from $\mathcal{B}$, then

$$
E\left(\bigcup_{n=1}^{\infty} X_{n}\right)=\sum_{n=1}^{\infty} E\left(X_{n}\right) .
$$


Then the spectral theorem states that for any normal operator $T$ there is a unique spectral measure $E^{T}$ on the Borel subsets of $\sigma(T)$ such that

$$
T=\int z d E^{T}(z) \text {. }
$$

This, at least in theory, completely describes the structure of a normal operator.

As an important consequence, it is now possible to completely classify normal operators up to unitary equivalence. Recall that two operators $T$, $S \in B(\mathcal{H})$ are unitarily equivalent if there exists $U \in U(\mathcal{H})$ such that $T=U S U^{-1}=U S U^{*}$. From results of multiplicity theory, developed based on the spectral theorem, for each normal operator $T$ there is a canonical sequence $x_{0}, x_{1}, \ldots \in \mathcal{H}$ such that the sequence of measures

$$
\mu_{n}^{T}=\left\langle E^{T}(\cdot) x_{n}, x_{n}\right\rangle,
$$

considered up to measure equivalence, gives a complete invariant for $T$ up to unitary equivalence. In other words, two normal operators $T$ and $S$ are unitarily equivalent if and only if, for all $n \in \mathbb{N}, \mu_{n}^{T}$ and $\mu_{n}^{S}$ are absolutely continuous with respect to each other.

The spectral theorem is usually considered the centerpiece of spectral theory, with the classification as one of the most important applications. For the purpose of generalizing the spectral theory, we will see that there is no obvious candidate for the spectral theorem, and so the classification becomes a natural subject of study. In particular, we do not want to hint that the spectral theory is equivalent to the classification, but our discussions will concentrate on the classification problem since the question appears natural to us.

Difficulties arise quickly when one tries to extend the above spectral theory to all bounded linear operators. The spectral theorem is obviously false since an integral with respect to a spectral measure always gives a normal operator.

The classification up to unitary equivalence, however, is not obviously false for all bounded linear operators. Imagine a statement saying that two bounded linear operators $T$ and $S$ are unitarily equivalent if and only if some sequences of measures obtained from $T$ and $S$, respectively, by some canonical means are absolutely continuous with respect to each other. In other words, some sequence of measures would completely determine the unitary equivalence type of the operator. This would be consistent in spirit with what a spectral theory is expected to achieve.

Still, this has not been done and the question is somewhat vague. We are now ready to consider some rigorous mathematical statements and give some results. To do this, we take advantage of the descriptive set theory of equivalence relations developed in the last two decades.

\section{Spectral Theory from the Point of View of Descriptive Set Theory}

Our first task is to turn the collection of our objects of study into a Polish space, that is, a separable, completely metrizable topological space. For this, let $B_{1}(\mathcal{H})$ be the space of all bounded linear operators on $\mathcal{H}$ with norm $\leq 1$. The operators in $B_{1}(\mathcal{H})$ are also called contractive. There are several Polish topologies on $B_{1}(\mathcal{H})$, the most well known being the strong operator topology (SOT) and the weak operator topology (WOT). For $T_{n}, T \in B_{1}(\mathcal{H})$, $T_{n} \rightarrow T$ in the SOT if $T_{n} x \rightarrow T x$ for all $x \in \mathcal{H}$, and $T_{n} \rightarrow T$ in the WOT if $\left\langle T_{n} x, y\right\rangle \rightarrow\langle T x, y\rangle$ for all $x, y \in \mathcal{H}$. Both topologies generate the same Borel structure. It is not obvious which topology is more advantageous since the multiplication (composition) operation on $B_{1}(\mathcal{H})$ is continuous in the SOT but not in the WOT and, on the other hand, the adjoint operation is continuous in the WOT but not in the SOT. For both the multiplication and the adjoint operations to be continuous, we consider the strong-star topology. For $T_{n}, T \in B_{1}(\mathcal{H}), T_{n} \rightarrow T$ in the strong-star topology if both $T_{n} \rightarrow T$ and $T_{n}^{*} \rightarrow T^{*}$ in the SOT. For our purposes we regard the strong-star topology to be the default Polish topology on $B_{1}(\mathcal{H})$.

Let $S_{1}(\mathcal{H})=S(\mathcal{H}) \cap B_{1}(\mathcal{H})$. Then obviously the strong-star topology coincides with the SOT on $S_{1}(\mathcal{H}) . S_{1}(\mathcal{H})$ is a closed subspace of $B_{1}(\mathcal{H})$ in the strong-star topology (in fact in either of the Polish topologies); hence it is itself a Polish space.

The WOT, the SOT, and the strong-star topology all coincide on $U(\mathcal{H})$, which is a closed subspace of $B_{1}(\mathcal{H})$ with the strong-star topology. Moreover, $U(\mathcal{H})$ is a topological group with multiplication as the group operation, and we refer to $U(\mathcal{H})$ as a Polish group since its underlying topology is Polish. $U(\mathcal{H})$ acts on $B_{1}(\mathcal{H})$ by conjugation, with the orbit equivalence relation representing the unitary equivalence. $S_{1}(\mathcal{H})$ is a closed invariant subspace for this action.

Finally, let $N_{1}(\mathcal{H})=N(\mathcal{H}) \cap B_{1}(\mathcal{H})$. Then the strong-star topology on $N_{1}(\mathcal{H})$ is again Polish. $N_{1}(\mathcal{H})$ is an invariant subspace for the conjugacy action of $U(\mathcal{H})$ on $B_{1}(\mathcal{H})$.

As noted above, understanding the unitary equivalence for all bounded linear operators comes fairly close to establishing a spectral theory for these operators. This unitary equivalence is in turn represented by the orbit equivalence relation of the conjugacy action of $U(\mathcal{H})$ on $B_{1}(\mathcal{H})$, which is now a continuous action of a Polish group on a Polish space.

Next we take a closer look at what the spectral theory of normal operators entails. This will help us identify some mathematical statements for investigation about all bounded linear operators. 
We claim that what the spectral theory of normal operators achieves is a reduction.

Let $\mathcal{E}, \mathcal{F}$ be equivalence relations on Polish spaces $\mathcal{X}, \mathcal{Y}$, respectively. A reduction from $\mathcal{E}$ to $\mathcal{F}$ is a function $f: \mathcal{X} \rightarrow y$ such that, for all $x_{1}, x_{2} \in \mathcal{X}$,

$$
x_{1} \mathcal{E} x_{2} \Longleftrightarrow f\left(x_{1}\right) \mathcal{F} f\left(x_{2}\right) .
$$

$f$ is called a Borel reduction if it is a Borel measurable function from $\mathcal{X}$ to $\mathcal{Y}$. We say that $\mathcal{E}$ is Borel reducible to $\mathcal{F}$, and denote it by $\mathcal{E} \leq_{B} \mathcal{F}$, if there exists a Borel reduction from $\mathcal{E}$ to $\mathcal{F}$.

With this definition, the classification of all normal operators up to unitary equivalence can be restated as one equivalence relation being reducible to another. On the left-hand side is the unitary equivalence for all normal operators, which is in turn represented by the orbit equivalence relation of the conjugacy action of $U(\mathcal{H})$ on $N_{1}(\mathcal{H})$. On the right-hand side is the equivalence of measures. To put this latter equivalence in perspective, we have to come up with a Polish space of measures.

Let $S$ be a compact metric space and let $\mathcal{B}$ be the collection of all Borel sets of $S$. Recall that a function $\mu: \mathcal{B} \rightarrow \mathbb{C}$ is a complex Borel measure on $S$ if $\mu(\varnothing)=0$ and for any collection $\left\{B_{n}\right\}_{n=1}^{\infty}$ of pairwise disjoint Borel sets,

$$
\mu\left(\bigcup_{n=1}^{\infty} B_{n}\right)=\sum_{n=1}^{\infty} \mu\left(B_{n}\right),
$$

where the series on the right converges absolutely. Let $M(S)$ be the space of all complex Borel measures on $S$, equipped with the topology generated by functions $\mu \mapsto \int f d \mu$, where $f \in C(X, \mathbb{C})$. Let

$$
\begin{aligned}
M_{1}(S)= & \left\{\mu \in M(S):\left|\int f d \mu\right| \leq 1\right. \\
& \text { for all } \left.f \in C(X, \mathbb{C}) \text { with }\|f\|_{\infty} \leq 1\right\} .
\end{aligned}
$$

Then $M_{1}(S)$ is a closed subspace of $M(S)$, and moreover, $M_{1}(S)$ is a compact metric space. Hence $M_{1}(S)$ is Polish.

The relations $\mu \ll v$ ( $\mu$ is absolutely continuous with respect to $\nu$ ) and $\mu \sim \nu$ ( $\mu$ and $\nu$ are equivalent) are Borel on $M_{1}(S)$ (meaning they are Borel subsets of $M_{1}(S) \times M_{1}(S)$ ). Moreover, it is easy to code a countable sequence of measures in $M_{1}(S)$ by a single measure in $M_{1}(S)$.

Putting all these together, the main theorem of multiplicity theory can be restated as:

The orbit equivalence relation of the conjugacy action of $U(\mathcal{H})$ on $N_{1}(\mathcal{H})$ is Borel reducible to the measure equivalence relation $\sim$ on $M_{1}(D)$, where $D$ is the unit disc on C.

The main addition in this restatement is the Borelness of the reduction function, which is not a trivial matter, but is straightforward to recover from the proofs of the theorems in spectral theory.
An obvious corollary of this statement is that the unitary equivalence of normal operators is a Borel equivalence relation because the measure equivalence relation is known to be Borel. Without spectral theory and multiplicity theory, this corollary would be by no means obvious.

Now to test the water with the possibility of a spectral theory for all bounded linear operators, it is natural to ask:

Is the orbit equivalence relation of the conjugacy action of $U(\mathcal{H})$ on $B_{1}(\mathcal{H})$ Borel?

If the answer turned out to be no, then it would explain why a spectral theory had not been developed, and one could even argue that this was enough evidence to conclude that there would never be a spectral theory.

The reason we stated at the beginning of this article that there might be a spectral theory, therefore, is that the answer to the above question is yes.

\section{What Does It Take to Prove This?}

We give a sketch of the proof below.

First note that every bounded linear operator $T \in B(\mathcal{H})$ can be written in the form $T=A^{T}+i B^{T}$, where $A^{T}, B^{T} \in S(\mathcal{H})$; in fact

$$
A^{T}=\frac{1}{2}\left(T+T^{*}\right), \quad B^{T}=\frac{1}{2 i}\left(T-T^{*}\right) .
$$

Moreover, if $S=U T U^{-1}$ for some $U \in U(\mathcal{H})$, then $A^{S}=U A^{T} U^{-1}$ and $B^{S}=U B^{T} U^{-1}$. This simple observation implies that the mapping $T \mapsto\left(A^{T}, B^{T}\right)$ is a reduction from the orbit equivalence relation of the conjugacy action of $U(\mathcal{H})$ on $B_{1}(\mathcal{H})$ to the orbit equivalence relation of the conjugacy action of $U(\mathcal{H})$ on the product space $S_{1}(\mathcal{H}) \times S_{1}(\mathcal{H})$. The reduction map is actually continuous. Thus, to see that the former equivalence relation is Borel, it suffices to investigate the diagonal action of $U(\mathcal{H})$ on $S_{1}(\mathcal{H}) \times S_{1}(\mathcal{H})$ and show that its orbit equivalence relation is Borel.

The diagonal actions of Polish groups on product Polish spaces and the Borelness of the orbit equivalence relation are the main objects of study in [2]. The next step of the proof is to cite one of the main results of [2]. Before doing this, we will need to recall some definitions. For any Polish space $\mathcal{X}$ the Effros Borel space $\mathcal{F}(\mathcal{X})$ is the space of all nonempty closed subsets of $X$, equipped with the Borel structure generated by basic Borel sets of the form

$$
\{F \in \mathcal{F}(\chi): F \cap O \neq \varnothing\}
$$

where $O$ is an open subset of $\chi$. This space was first defined by Effros in 1965 in [3]. He proved that $\mathcal{F}(\mathcal{X})$ is a standard Borel space, that is, there is an underlying Polish topology generating the Borel structure. 
Suppose $G$ is a Polish group, $Z$ is a Polish space, and $a: G \times Z \rightarrow Z$ is a Borel measurable action of $G$ on $Z$. For any $z \in Z$, the stabilizer of $z$ is

$$
G_{Z}=\{g \in G: a(g, z)=z\} .
$$

$G_{Z}$ is always a closed subgroup of $G$. We also refer to the map $Z \mapsto G_{Z}$ as the stabilizer map. The stabilizer map is therefore a map from $Z$ into $\mathcal{F}(G)$. Let $E_{a}$ denote the orbit equivalence relation of the action $a$. A well-known theorem of Becker and Kechris [1] states that $E_{a}$ is Borel if and only if the stabilizer map is Borel.

The main theorem we cite from [2], Theorem 4.4 , is a refinement of the above theorem of Becker and Kechris. The following is a simplified version tailored for our purpose.

Let $G$ be a Polish group and let $S$ be a Borel subset of $\mathcal{F}(G)$ that is closed under conjugation. Then the following are equivalent:

(i) The intersection operation $(X, Y) \mapsto X \cap$ $Y$ from $S \times S$ to $\mathcal{F}(G)$ is Borel.

(ii) Given any Polish space $Z$ and Borel measurable action $a: G \times Z \rightarrow Z$ of $G$ on $Z$, let $E_{a}$ be the orbit equivalence relation of the action $a$. Let $b: G \times(Z \times Z) \rightarrow$ $(Z \times Z)$ be the diagonal action:

$$
b(g,(u, v))=(a(g, u), a(g, v)) \text {, }
$$

for $g \in G, u, v \in Z$. Let $E_{b}$ be the orbit equivalence relation of the action $b$. Assume $G_{z} \in S$ for every $z \in Z$. If $E_{a}$ is Borel, then $E_{b}$ is Borel.

To apply the theorem in our context, let $G=$ $U(\mathcal{H}), Z=S_{1}(\mathcal{H})$, and we have yet to suitably define the collection $S$. Note that the Borelness assumption on $E_{a}$ is given by the spectral theory for self adjoint operators, which is a special case of the spectral theory for normal operators.

To define the collection $S$ we need the notion of von Neumann algebras. A subspace of $B(\mathcal{H})$ is called a *-subalgebra if it is closed under multiplication and the adjoint operations. A $*$ subalgebra of $B(\mathcal{H})$ is called a von Neumann algebra if it is closed in the WOT (or equivalently, closed in the SOT). If $C \subseteq S(\mathcal{H})$ is any subset, the commutant

$$
C^{\prime}=\{T \in B(\mathcal{H}): T S=S T \text { for any } S \in C\}
$$

is a von Neumann algebra. It is easy to see that, if $S \in S_{1}(\mathcal{H})$, then

$$
\{S\}^{\prime} \cap U(\mathcal{H})=\left\{U \in U(\mathcal{H}): S=U S U^{-1}\right\},
$$

which is the stabilizer $U(\mathcal{H})_{S}$ for the conjugacy action of $U(\mathcal{H})$ on $S_{1}(\mathcal{H})$.

We now define the collection $S$ to be $\operatorname{vNA}(U(\mathcal{H}))$, the collection of all sets of the form $\mathcal{A} \cap U(\mathcal{H})$, where $\mathcal{A}$ is a von Neumann algebra. We will prove the Borelness of $\operatorname{vNA}(U(\mathcal{H}))$ (as a subset of $\mathcal{F}(U(\mathcal{H})))$ as well as that of the intersection operation in the final step below.

Let $\mathcal{F}^{*}$ denote the Effros Borel space of all nonempty subsets of $B_{1}(\mathcal{H})$ that are closed in the strong-star topology. We will also consider $B_{1}(\mathcal{H})$ with the WOT, which makes $B_{1}(\mathcal{H})$ a compact space. Let $\mathcal{F}^{w}$ denote the Effros Borel space of all nonempty subsets of $B_{1}(\mathcal{H})$ that are closed in the WOT. Since all strong-star open sets are WOT $K_{\sigma}$ on $B_{1}(\mathcal{H})$, the identity map Id $: \mathcal{F}^{w} \rightarrow \mathcal{F}^{*}$ is a Borel injection. We will use this tacitly in the computations below.

Let $\operatorname{vNA}\left(B_{1}(\mathcal{H})\right)$ be the collection of all sets of the form $\mathcal{A} \cap B_{1}(\mathcal{H})$, where $\mathcal{A}$ is a von Neumann algebra. We claim that $\operatorname{vNA}\left(B_{1}(\mathcal{H})\right)$ is a Borel subset of $\mathcal{F}^{w}$. To see this, let $\left\{\xi_{m}: m \in \mathbb{N}\right\}$ be a dense subset of the unit ball $B$ of $\mathcal{H}$. Fix a sequence of Borel selector maps $s_{n}: \mathcal{F}^{*} \rightarrow B_{1}(\mathcal{H})$ such that, for each nonempty $F \in \mathcal{F}^{*},\left\{s_{n}(F): n \in \mathbb{N}\right\}$ is dense in $F$ in the strong-star topology. Such maps exist by the Borel selection theorem of Kuratowski and Ryll-Nardzewski (cf. Theorem 12.13 of [6]). Keep in mind that both multiplication and the adjoint operations are continuous on $B_{1}(\mathcal{H})$ with the strong-star topology. We can now calculate, for any $F \in \mathcal{F}^{w}$, that $F \in \operatorname{vNA}\left(B_{1}(\mathcal{H})\right)$ if and only if

$$
\begin{aligned}
& \forall n \forall q \in \mathbb{Q}\left[\forall m\left(q\left\|s_{n}(F) \xi_{m}\right\| \leq 1\right) \Rightarrow q s_{n}(F) \in F\right] \\
& \& \forall k \forall l\left[\frac{1}{2}\left(s_{k}(F)+s_{l}(F)\right) \in F \& s_{k}(F) s_{l}(F) \in F\right] \\
& \& \forall n\left[s_{n}(F)^{*} \in F\right] .
\end{aligned}
$$

Since $B_{1}(\mathcal{H})$ is compact in the WOT, the intersection operation on $\mathcal{F}^{w}$ is Borel. It follows that the intersection operation on $\operatorname{vNA}\left(B_{1}(\mathcal{H})\right)$ is Borel. To prove the Borelness of the intersection operation on $\operatorname{vNA}(U(\mathcal{H}))$, as well as to show that $\operatorname{vNA}(U(\mathcal{H}))$ itself is a Borel subset of $\mathcal{F}(U(\mathcal{H}))$, we only need to note that there is a Borel bijection between $\operatorname{vNA}\left(B_{1}(\mathcal{H})\right)$ and $\operatorname{vNA}(U(\mathcal{H}))$ that preserves the intersection operation. The obvious candidate $F \mapsto F \cap U(\mathcal{H})$ works. In fact, since the map $T \mapsto \exp \left(2 \pi i B^{T}\right)$ from $B_{1}(\mathcal{H})$ to $U(\mathcal{H})$ is continuous with respect to the strong-star topology, we have that if $F \in \operatorname{vNA}\left(B_{1}(\mathcal{H})\right)$, then $F \cap U(\mathcal{H})$ is the closure of

$$
\left\{\exp \left(2 \pi i B^{S_{n}(F)}\right): n \in \mathbb{N}\right\} .
$$

This finishes the proof of our main claim of this article, which is that the unitary equivalence for all bounded linear operators is a Borel equivalence relation.

\section{A Better Theorem}

Descriptive set theory can be viewed as a complexity theory for sets of real numbers. By coding objects as elements of Polish spaces, this complexity theory can be extended to sets which occur on higher levels in the cumulative hierarchy of sets 
(the cumulative hierarchy is another important contribution of von Neumann to mathematics). The notion of Borel reducibility gives rise to a complexity theory of equivalence relations on Polish spaces. If $\mathcal{E}$ is Borel reducible to $\mathcal{F}$, then intuitively we regard $\mathcal{E}$ as being no more complex than $\mathcal{F}$ because questions about $\mathcal{E}$ can be answered by resolving questions about $\mathcal{F}$ via the reduction map. By Borel reducibility one can also speak of the essential complexity of a Borel equivalence relation in the usual Borel hierarchy, as follows.

First recall that the $\sigma$-algebra of all Borel sets on a Polish space can be stratified into classes $\Sigma_{\alpha}^{0}, \Pi_{\alpha}^{0}$, and $\Delta_{\alpha}^{0}$ for countable ordinals $\alpha \geq 1$. On each Polish space $\mathcal{X}$, these classes are defined by a transfinite induction:

(i) $\Sigma_{1}^{0}$ is the collection of all open subsets of $x$

(ii) for each countable ordinal $\alpha \geq 1, \Pi_{\alpha}^{0}$ is the collection of all subsets of $\chi$ whose complement is in $\Sigma_{\alpha}^{0}$,

(iii) for each $\alpha>1, \Sigma_{\alpha}^{0}$ is the collection of all subsets of $\mathcal{X}$ of the form $\bigcup_{n=1}^{\infty} S_{n}$, where each $S_{n}$ is in $\Pi_{\beta_{n}}^{0}$ for some $\beta_{n}<\alpha$,

(iv) for each $\alpha \geq 1, \Delta_{\alpha}^{0}=\Sigma_{\alpha}^{0} \cap \Pi_{\alpha}^{0}$.

For an uncountable Polish space $\chi$, the Borel hierarchy does not collapse; that is, if $\alpha>\beta$, then each of the classes on the $\beta$-th level is a proper subset of any of the classes on the $\alpha$-th level.

Let $\mathcal{E}$ be a Borel equivalence relation on a Polish space $\mathcal{X}$. Let $\alpha \geq 1$ be a countable ordinal. We say that $\mathcal{E}$ is essentially $\Pi_{\alpha}^{0}$ if there is a $\Pi_{\alpha}^{0}$ equivalence relation $\mathcal{F}$ on a Polish space $\mathcal{y}$ so that $\mathcal{E}$ is Borel reducible to $\mathcal{F}$. In a similar fashion one can define the notion of essential $\Sigma_{\alpha}^{0}$ and essential $\Delta_{\alpha}^{0}$. Of course, if the equivalence relation $\mathcal{E}$ itself is $\Pi_{\alpha}^{0}$, then it is essentially $\Pi_{\alpha}^{0}$.

With this notion in hand let us take a closer look at the unitary equivalence of bounded linear operators. Again we start from the normal operators. As we noted before, spectral theory and multiplicity theory give that the unitary equivalence of normal operators is Borel reducible to the measure equivalence. By a straightforward computation, one can see that the measure equivalence is a $\Pi_{3}^{0}$ equivalence relation on $M_{1}(D)$. Thus we conclude that the unitary equivalence of normal operators is essentially $\Pi_{3}^{0}$.

If the unitary equivalence for all bounded linear operators turns out to be an essentially $\Pi_{3}^{0}$ equivalence relation, then we would be more confident about the existence of a new spectral theory.

The proof we demonstrated above for the Borelness of the unitary equivalence of all bounded linear operators, however, does not give an essential class for the equivalence relation. It does not even allow us to compute the Borel rank of the equivalence relation because there is no levelby-level correspondence of the Borelness of the intersection operation with that of the orbit equivalence relation of the diagonal action.

The punch line, as the reader may have suspected, is that, yes, the unitary equivalence for all bounded linear operators is essentially $\Pi_{3}^{0}$, and this is the reason we stated at the beginning of the article that a new spectral theory is likely to exist.

Strangely enough, the story of this result goes back to another paper [4] of Effros published in 1965. The main result of that paper is that the commutant, join, and intersection operations on the space vNA $(B(\mathcal{H}))$ are Borel. But the relevance of the paper in recent research comes from a problem that Effros stated on unitary representations.

Let $\Gamma$ be a countable discrete group. A unitary representation of $\Gamma$ is a homomorphism $\rho: \Gamma \rightarrow U(\mathcal{H})$. Let $\operatorname{Rep}(\Gamma, \mathcal{H})$ be the space of all unitary representations of $\Gamma$. Since each unitary representation can be viewed as an element of $U(\mathcal{H})^{\Gamma}$, the space $\operatorname{Rep}(\Gamma, \mathcal{H})$ can be viewed as a subspace of $U(\mathcal{H})^{\Gamma}$. Equipping $U(\mathcal{H})^{\Gamma}$ with the product topology, $\operatorname{Rep}(\Gamma, \mathcal{H})$ is a closed subspace, hence is Polish. Two representations $\rho$ and $\pi$ of $\Gamma$ are unitarily equivalent if there exists $U \in U(\mathcal{H})$ such that for all $g \in \Gamma$,

$$
\rho(g)=U \pi(g) U^{-1} .
$$

Effros asked if the unitary equivalence of all unitary representations of $\Gamma$ is a Borel equivalence relation.

This was recently given a positive answer by Hjorth and Törnquist [5]. In fact, they were able to determine the Borel rank of these equivalence relations:

For any countable discrete group $\Gamma$, the unitary equivalence relation on $\operatorname{Rep}(\Gamma, \mathcal{H})$ is $\Pi_{3}^{0}$.

As a direct corollary, the orbit equivalence relation of the diagonal conjugacy action of $U(\mathcal{H})$ on $U(\mathcal{H}) \times U(\mathcal{H})$ is also $\Pi_{3}^{0}$. To see this, consider the reduction map from $U(\mathcal{H}) \times U(\mathcal{H})$ to $\operatorname{Rep}\left(\mathbb{F}_{2}, \mathcal{H}\right)$, where $\mathbb{F}_{2}=\langle a, b\rangle$ is the free group with two generators $a$ and $b$, given by $(S, T) \mapsto \rho$, where

$$
\rho(w(a, b))=w(S, T)
$$

for any group word $w(a, b) \in \mathbb{F}_{2}$. Note that this map is continuous.

Finally, it suffices to see that the unitary equivalence of all bounded linear operators is Borel reducible to the above diagonal orbit equivalence relation. Earlier in this article we mentioned that it is Borel reducible to the orbit equivalence relation of the diagonal conjugacy action of $U(\mathcal{H})$ on $S_{1}(\mathcal{H}) \times S_{1}(\mathcal{H})$. Now it suffices to note that this reduction can be composed with another reduction from $S_{1}(\mathcal{H})$ to $U(\mathcal{H})$ via the map $T \mapsto \exp (2 \pi i T)$. 
It is easy to see that all the reductions used in the composition above are continuous, which implies that the unitary equivalence of all bounded linear operators is itself a $\Pi_{3}^{0}$ equivalence relation.

With this result we are one step closer to reaching a spectral theory. One can extract from [5] the functional analytical machinery that is needed to effectively decide when a pair of bounded linear operators are unitarily equivalent. However, what is expected from a spectral theory is a method to extract all important structural information from a single operator, so that a classification can be performed by comparing this information extracted separately from different operators.

\section{What's Next?}

The next rigorous question that can be asked from the point of view of descriptive set theory is the following:

Is the unitary equivalence of all bounded linear operators Borel reducible to the measure equivalence?

As we speculated before, if the answer turns out to be positive, then it should be possible to extract a new spectral theory from its proof.

Of course, the ultimate solution to the problem is likely to need all the techniques of functional analysis, especially operator theory. The story told in this article confirms again that all of mathematics is deeply connected, and we hope the reader is as excited as we are by these connections.

\section{References}

[1] H. Becker and A. S. Kechris, The Descriptive Set Theory of Polish Group Actions, London Mathematical Society Lecture Note Series, no. 232, Cambridge University Press, 1996.

[2] L. DiNG and S. GAO, Diagonal actions and Borel equivalence relations, The Journal of Symbolic Logic 71 (2006), no. 4, 1081-1096.

[3] E. G. EFfros, Convergence of closed subsets in a topological space, Proceedings of the American Mathematical Society 16 (1965), 929-931.

[4] _ The Borel space of von Neumann algebras on a separable Hilbert space, Pacific Journal of Mathematics 15 (1965), 1153-1164.

[5] G. HJORTH and A. TöRNQUIST, The conjugacy relations on unitary representations, Mathematical Research Letters 19 (2012), no. 3, 525-535.

[6] A. S. Kechris, Classical Descriptive Set Theory, Graduate Texts in Mathematics, Vol. 156, Springer-Verlag, New York, 1995.

\section{Mathematics Journals}

\section{FROM CAMBRIDGE}

High quality pure and applied mathematics research:

- The ANZIAM journal

- Mathematical Proceedings of the Cambridge Philosophical Society

- Compositio Mathematica

- Proceedings of the Royal Society of Edinburgh, Section A: Mathematics

- Journal of the Institute of Mathematics of Jussieu
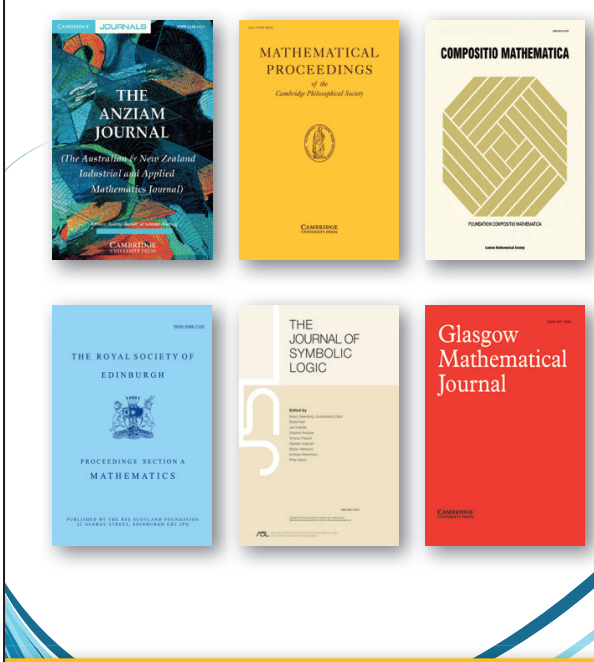

\section{FREE} ACCESS OFFER

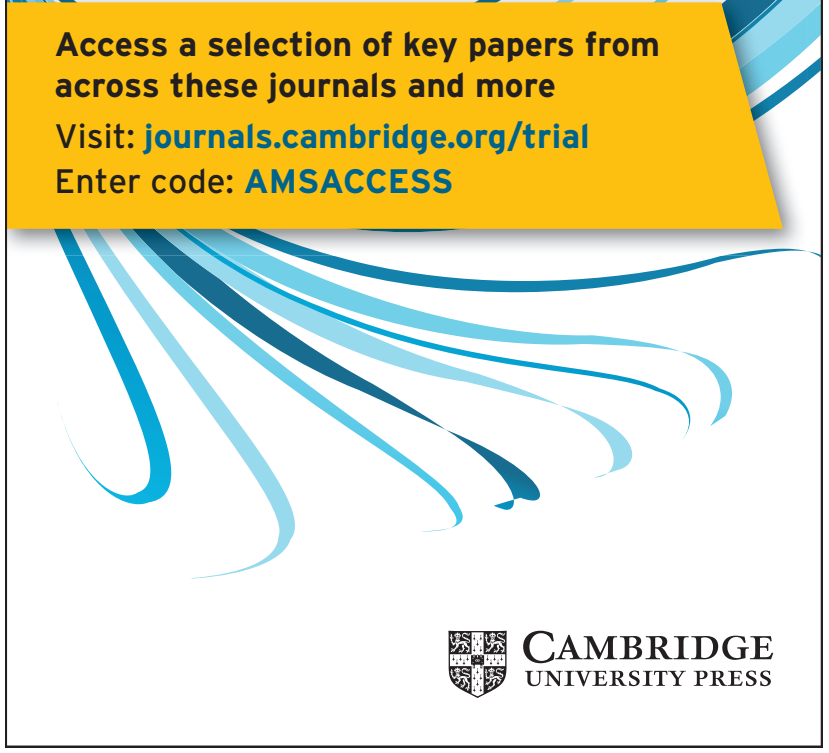

